\title{
TRANSLATION FOLIATIONS OF CODIMENSION ONE ON COMPACT AFFINE MANIFOLDS
}

\author{
FRANCISCO JAVIER TURIEL \\ Geometría y Topología, Facultad de Ciencias Ap. 59, Universidad de Málaga \\ 29080 Málaga, Spain \\ E-mail:turiel@ccuma.sci.uma.es
}

\begin{abstract}
Consider two foliations $\mathcal{F}_{1}$ and $\mathcal{F}_{2}$, of dimension one and codimension one respectively, on a compact connected affine manifold $(M, \nabla)$. Suppose that $\nabla_{T \mathcal{F}_{1}} T \mathcal{F}_{2} \subset T \mathcal{F}_{2}$; $\nabla_{T \mathcal{F}_{2}} T \mathcal{F}_{1} \subset T \mathcal{F}_{1}$ and $T M=T \mathcal{F}_{1} \oplus T \mathcal{F}_{2}$. In this paper we show that either $\mathcal{F}_{2}$ is given by a fibration over $S^{1}$, and then $\mathcal{F}_{1}$ has a great degree of freedom, or the trace of $\mathcal{F}_{1}$ is given by a few number of types of curves which are completely described. Moreover we prove that $\mathcal{F}_{2}$ has a transverse affine structure.
\end{abstract}

Introduction. We work in the $C^{\infty}$ category.

Consider a compact connected affine manifold $(M, \nabla)$, i.e. $\nabla$ is a connection whose torsion and curvature vanish, of dimension $n$ equipped with a finite family of foliations $\mathcal{F}_{1}, \ldots, \mathcal{F}_{k}$. We will say that $\mathcal{F}_{1}, \ldots, \mathcal{F}_{k}$ are translation foliations (T.F.) if for any $1 \leq$ $i<j \leq k$ we have $\nabla_{T \mathcal{F}_{i}} T \mathcal{F}_{j} \subset T \mathcal{F}_{j}$ and $\nabla_{T \mathcal{F}_{j}} T \mathcal{F}_{i} \subset T \mathcal{F}_{i}$, where $T \mathcal{F}_{i}$ and $T \mathcal{F}_{j}$ are the vector subbundles of tangent vectors to the leaves of $\mathcal{F}_{i}$ and $\mathcal{F}_{j}$ respectively.

This kind of structures appears, in a natural way, when we consider a bilagrangian fibration $\pi:\left(N, \omega, \omega_{1}\right) \rightarrow M$ whose fibres are tori $\mathbf{T}^{n}$. Then $M$ is endowed with two integer affine structure $\mathcal{A}$ and $\mathcal{A}_{1}$ and a $(1,1)$ tensor field $J$ which transforms $\mathcal{A}$ on $\mathcal{A}_{1}$. The eigenspaces of $J$ give rise to a family of translation foliations $\mathcal{F}_{1}, \ldots, \mathcal{F}_{k}$ which are transverse (often with some singularities, see [1]).

Another example is given by Veronese webs when they are affine. Veronese webs have been introduced by Gelfand and Zakarevich for studying the bihamiltonian systems of odd dimension (see [5]).

Translation foliations on surfaces have been studied by Darboux (see [3]).

Here we will consider the case of two translation foliations $\mathcal{F}_{1}$ and $\mathcal{F}_{2}$ which are transverse, i.e. $T M=T \mathcal{F}_{1} \oplus T \mathcal{F}_{2}$, such that $\operatorname{dim} \mathcal{F}_{1}=\operatorname{codim} \mathcal{F}_{2}=1$. For the sake of

1991 Mathematics Subject Classification: Primary 53C12; Secondary 53A15.

Supported by DGICYT under grant PB94-1485.

The paper is in final form and no version of it will be published elsewhere. 
simplicity $\mathcal{F}_{1}$ and $\mathcal{F}_{2}$ shall be assumed orientable. This last condition is always satisfied by taking a finite covering if necessary. Let us remark that $\mathcal{F}_{1}$ and $\mathcal{F}_{2}$ are transversely orientable as well.

In affine coordinates the property of translation is equivalent to the following one: consider an open set $A_{1}$ of a leaf $\mathcal{F}_{1}$, an open set $A_{2}$ of a leaf of $\mathcal{F}_{2}$ and a point $p \in A_{1} \cap A_{2}$; given $q_{1} \in A_{1}$ and $q_{2} \in A_{2}$ then $A_{1}+q_{2}-p$ is an open set of the leaf of $\mathcal{F}_{1}$ passing through $q_{2}$, and $A_{2}+q_{1}-p$ is an open set of the leaf of $\mathcal{F}_{2}$ passing through $q_{1}$ (obviously where it has a meaning). In other words, around $p$ foliations $\mathcal{F}_{1}$ and $\mathcal{F}_{2}$ are completely determined by the leaves of $\mathcal{F}_{1}$ and $\mathcal{F}_{2}$ passing through this point.

In this paper we show that $\mathcal{F}_{2}$ always has a transverse affine structure. Besides one of the three following possibilities holds:

1) Foliation $\mathcal{F}_{2}$ is given by a fibration over $S^{1}$. Then $\mathcal{F}_{1}$ has a great degree of freedom. Nevertheless $\mathcal{F}_{1}$ is spanned by an $\mathcal{F}_{2}$-foliate and $\mathcal{F}_{2}$-parallel vector field.

2) Foliation $\mathcal{F}_{2}$ has trivial holonomy and all its leaves are dense. Then there exist real numbers $a_{0}, \ldots, a_{k-1}$ and a non-singular vector field $X$, tangent to $\mathcal{F}_{1}$, which is both $\mathcal{F}_{2}$-parallel and $\mathcal{F}_{2}$-foliate, such that $\nabla_{X}^{k} X=\sum_{j=0}^{k-1} a_{j} \nabla_{X}^{j} X$. Moreover $k \leq \operatorname{rank} M+1$.

Therefore, in affine coordinates, $\mathcal{F}_{1}$ is described by a curve $\gamma(t)$ which is a solution of the equation $\gamma^{(k+1)}=\sum_{j=1}^{k} a_{j-1} \gamma^{(j)}$.

3) Foliation $\mathcal{F}_{2}$ has non-trivial holonomy. Then $\mathcal{F}_{2}$ has a finite number of minimal sets, all of them with non-trivial holonomy, and there exist natural numbers $r_{1}=1<$ $r_{2}<\ldots<r_{k}$ such that, around each point, we may find affine coordinates on which the polynomial curve $\gamma(t)=\left(t, t^{r_{2}}, \ldots, t^{r_{k}}, 0, \ldots, 0\right)$ describes $\mathcal{F}_{1}$.

\section{Moreover:}

If $r_{k}>k$ then all the non-compact leaves of $\mathcal{F}_{2}$ have trivial holonomy; on the other hand the compact ones are just the only minimal sets.

If the affine manifold $(M, \nabla)$ is complete then $r_{j}=j, j=1, \ldots, k$ and all the leaves of $\mathcal{F}_{2}$ are dense.

\section{Examples.}

(a) Consider two imbeddings $f_{1}: S^{1} \rightarrow \mathbf{T}^{n} ; f_{2}: \mathbf{T}^{n-1} \rightarrow \mathbf{T}^{n}$, where $\mathbf{T}^{k}$ is the torus of dimension $k$, and a point $p \in \mathbf{T}^{n}$. Assume that:

(I) There exists $\alpha_{0} \in S^{1}$ and $\beta_{0} \in \mathbf{T}^{n-1}$ such that $p=f_{1}\left(\alpha_{0}\right)=f_{2}\left(\beta_{0}\right)$ and $\left\{f_{1 *} \pi_{1}\left(S^{1}, \alpha_{0}\right), f_{2 *} \pi_{1}\left(\mathbf{T}^{n-1}, \beta_{0}\right)\right\}$ spans $\pi_{1}\left(\mathbf{T}^{n}, p\right)$.

(II) For any $(\alpha, \beta) \in S^{1} \times \mathbf{T}^{n-1}$ the subspaces $f_{1 *}\left(T_{\alpha} S^{1}\right)$ and $f_{2 *}\left(T_{\beta} \mathbf{T}^{n-1}\right)$, after being carried to $0 \in \mathbf{T}^{n}$ by means of the canonical connection, are transverse.

Then the map $F:(\alpha, \beta) \in S^{1} \times \mathbf{T}^{n-1} \rightarrow f_{1}(\alpha)+f_{2}(\beta) \in \mathbf{T}^{n}$ is a diffeomorphism and the foliations $\mathcal{F}_{1}$ and $\mathcal{F}_{2}$, defined by the submersions $\pi_{2} \circ F^{-1}: \mathbf{T}^{n} \rightarrow \mathbf{T}^{n-1}$ and $\pi_{1} \circ F^{-1}: \mathbf{T}^{n} \rightarrow \mathbf{S}^{1}$, are T.F.

(b) On $\widetilde{M}=\mathbf{R} \times \mathbf{R}^{+}$we consider the equivalence relation $x \mathcal{R} y$ if and only if $y_{1}=$ $x_{1}+k_{1}$ and $y_{2}=\exp \left(k_{2}\right) x_{2}$ where $k_{1}, k_{2} \in \mathbf{Z}$, and the foliations $\widetilde{\mathcal{F}}_{1}$, given by the curves $x_{2} \exp \left(-x_{1}\right)=$ constant, and $\widetilde{\mathcal{F}}_{2}$, associated to vector field $\frac{\partial}{\partial x_{1}}$. Set $M=\widetilde{M} / \mathcal{R}$. By projecting $\widetilde{\mathcal{F}}_{1}$ and $\widetilde{\mathcal{F}}_{2}$ manifold $M$ is endowed with two foliations $\mathcal{F}_{1}$ and $\mathcal{F}_{2}$ which are 
T.F. with respect to the projected connection.

(c) Let $\left\{e_{1}, e_{2}\right\}$ be a basis of the Lie algebra of $\mathbf{T}^{2}$. Now equip $\mathbf{T}^{2}$ with the affine connection given by $\nabla_{e_{1}} e_{1}=e_{2} ; \nabla_{e_{i}} e_{j}=0$ otherwise. Then the foliations $\mathcal{F}_{1}$, associated to $e_{1}$, and $\mathcal{F}_{2}$, associated to $e_{2}$, are T.F. Moreover $\mathcal{F}_{1}$ is parabolic, i.e. around each point there exist affine coordinates on which the leaf of $\mathcal{F}_{1}$ passing through this point can be written $\left(t, t^{2}\right)$, and $\mathcal{F}_{2}$ is geodesic.

In Example (b) $\mathcal{F}_{2}$ is geodesic as well and the leaves of $\mathcal{F}_{1}$ are written $\left(t, a e^{t}\right)$ in suitable affine coordinates.

(d) Hopf structure. Given positive natural numbers $r_{2}, \ldots, r_{n}$, set $X=\frac{\partial}{\partial x_{1}}+\sum_{j=2}^{n} x_{1}^{r_{j}-1} \frac{\partial}{\partial x_{j}}$ and $Y=x_{1} \frac{\partial}{\partial x_{1}}+\sum_{j=2}^{n} r_{j} x_{j} \frac{\partial}{\partial x_{j}} ;$ then $[X, Y]=X$. On $\widetilde{M}=\mathbf{R}^{n}-\{0\}, n \geq 2$, the foliations $\widetilde{\mathcal{F}}_{1}$, associated to $X$, and $\widetilde{\mathcal{F}}_{2}$, defined by $d x_{1}=0$, are T.F. with respect to the canonical connection of $\mathbf{R}^{n}$. On the other hand they are preserved by the flow $\phi_{t}$ of $Y$.

On $\widetilde{M}$ we define the equivalence relation $x \mathcal{R} y$ if and only if $\phi_{\ell}(x)=y$ for some $\ell \in \mathbf{Z}$. As the vector field $Y$ is both affine and foliate the quotient manifold $M$, i.e. $S^{1} \times S^{n-1}$, is affine and the projected foliations $\mathcal{F}_{1}$ and $\mathcal{F}_{2}$ are T.F. Foliation $\mathcal{F}_{2}$ has non-trivial holonomy and, in suitable affine coordinates, each leaf of $\mathcal{F}_{1}$ is given by the curve $\left(t, t^{r_{2}}, \ldots, t^{r_{n}}\right)$.

Other foliations which are T.F. may be constructed in the same way. For example on $\mathbf{R}^{3}-\{0\}$ we can set $Y=2 x_{1} \frac{\partial}{\partial x_{1}}+4 x_{2} \frac{\partial}{\partial x_{2}}+x_{3} \frac{\partial}{\partial x_{3}}$ and consider the foliations $\widetilde{\mathcal{F}}_{1}$, given by $\frac{\partial}{\partial x_{1}}+\left(x_{1}+x_{3}^{2}\right) \frac{\partial}{\partial x_{2}}$, and $\widetilde{\mathcal{F}}_{2}$ associated to $\frac{\partial}{\partial x_{2}} ;-2 x_{3} \frac{\partial}{\partial x_{1}}+\frac{\partial}{\partial x_{3}}$.

(e) On $\mathbf{T}^{3}$ consider the affine connection obtained by setting $\nabla \frac{\partial}{\partial \alpha_{1}} \frac{\partial}{\partial \alpha_{1}}=\frac{\partial}{\partial \alpha_{3}}$; $\nabla_{\frac{\partial}{\partial \alpha_{2}}} \frac{\partial}{\partial \alpha_{2}}=2 \frac{\partial}{\partial \alpha_{3}} ; \nabla_{\frac{\partial}{\partial \alpha_{i}}} \frac{\partial}{\partial \alpha_{j}}=0$ otherwise, and the translation foliations $\mathcal{F}_{1}$ and $\mathcal{F}_{2}$ spanned by $\frac{\partial}{\partial \alpha_{1}}+2^{-\frac{1}{2}} \frac{\partial}{\partial \alpha_{2}}$ and $\frac{\partial}{\partial \alpha_{1}}-2^{-\frac{1}{2}} \frac{\partial}{\partial \alpha_{2}} ; \frac{\partial}{\partial \alpha_{3}}$ respectively. Now the affine structure is integer, $\mathcal{F}_{1}$ is parabolic and all the leaves of $\mathcal{F}_{2}$ are dense.

(f) Consider a compact connected affine manifold $(M, \nabla)$ equipped with two translation foliations $\mathcal{F}_{1}$ and $\mathcal{F}_{2}$, such that $\operatorname{dim} \mathcal{F}_{1}=\operatorname{codim} \mathcal{F}_{2}=1$ and $T \mathcal{F}_{1} \oplus T \mathcal{F}_{2}=T M$. Let $G$ be the group of affine diffeomorphisms of $(M, \nabla)$ which preserve $\mathcal{F}_{1}$ and $\mathcal{F}_{2}$. Consider a second compact connected affine manifold $W$. Assume that $W, \mathcal{F}_{1}$ and $\mathcal{F}_{2}$ are orientable. If $\widetilde{W}$ is the universal covering of $W$ then $\widetilde{W} \times M$ can be endowed with the translation foliations $\widetilde{\mathcal{F}}_{1}$ and $\widetilde{\mathcal{F}}_{2}$ given by $T \widetilde{\mathcal{F}}_{1}=\{0\} \times T \mathcal{F}_{1}$ and $T \widetilde{\mathcal{F}}_{2}=T W \times T \mathcal{F}_{2}$. Therefore, by suspending each morphism from $\pi_{1}(W)$ to $G$, we obtain a new example of translation foliations.

If $\left(M, \mathcal{F}_{1}, \mathcal{F}_{2}\right)$ is as in Example (d), then $Y$ gives rise to a vector field $Y^{\prime}$, on $M$, whose flow $\phi_{t}^{\prime}$ is included in $G$. Now by taking $W=S^{1}$ and $\phi_{b}^{\prime}$, where $b \notin \mathbf{Q}$, as image of a generator of $\pi_{1}\left(S^{1}\right)$, one constructs an example of translation foliations where the codimension one foliation only has one $(\operatorname{dim} M>2)$ or two $(\operatorname{dim} M=2)$ compact leaves. The other ones are locally dense (note that all the leaves of $\mathcal{F}_{2}$ were proper; obviously the number of compact leaves of this last foliation is the same as before).

(g) Given $B \in S L(\mathbf{Z}, 2)$ let $\varphi_{B}: \mathbf{T}^{2} \rightarrow \mathbf{T}^{2}$ be the associated isomorphism. Consider an element $A \in S L(\mathbf{Z}, 2)$ with two distinct positive real eigenvalues $\lambda_{1}, \lambda_{2}$. Let $\left\{d_{1}, d_{2}\right\}$ be a basis of the Lie algebra of $\mathbf{T}^{2}$ such that $\left(\varphi_{A}\right)_{*} d_{i}=\lambda_{i} d_{i}, i=1,2$. Now endow 
$\mathbf{T}^{4}=\mathbf{T}^{2} \times \mathbf{T}^{2}$ with the affine structure given by $\nabla_{e_{1}} e_{1}=e_{3} ; \nabla_{e_{i}} e_{j}=0$ otherwise, where $e_{1}=\left(d_{1}, 0\right), e_{2}=\left(d_{2}, 0\right), e_{3}=\left(0, d_{1}\right), e_{4}=\left(0, d_{2}\right)$. Let $\mathcal{F}_{1}$ and $\mathcal{F}_{2}$ the translation foliations spanned by $e_{1}$ and $\left\{e_{2}, e_{3}, e_{4}\right\}$ respectively.

By suspending the diffeomorphism $\phi=\left(\varphi_{A}, \varphi_{A^{2}}\right)$ as in Example (f) $\left(W=S^{1}\right)$ one constructs two translation foliations $\mathcal{F}^{\prime}{ }_{1}$ and $\mathcal{F}^{\prime}{ }_{2}$, on a compact affine 5 -manifold $M^{\prime}$, the first one parabolic and the second one with non-trivial holonomy and dense leaves. Note that the affine manifold $M^{\prime}$ is complete (see Corollary 5.1).

2. The polynomial points of $\mathcal{F}_{1}$. If each leaf of $\mathcal{F}_{2}$ is compact and its holonomy is trivial, i.e. if $\mathcal{F}_{2}$ is given by a fibration over $S^{1}$, the leaves of $\mathcal{F}_{1}$ have a great degree of freedom as Example (a) shows. Nevertheless they are the orbits of a non-singular vector field $X$ which is both $\mathcal{F}_{2}$-foliate and $\mathcal{F}_{2}$-parallel. Indeed, as $\mathcal{F}_{1}$ and $\mathcal{F}_{2}$ are T.F. all $\mathcal{F}_{2}$-foliate vector field is parallel. Conversely if a non-singular $\mathcal{F}_{2}$-foliate vector field $X$, tangent to $\mathcal{F}_{1}$, is $\mathcal{F}_{2}$-parallel then $\mathcal{F}_{1}$ and $\mathcal{F}_{2}$ are T.F. (even if the leaves of $\mathcal{F}_{2}$ are dense). Obviously $\mathcal{F}_{2}$ has transverse affine structures.

From now on we will suppose, if necessary, that $\mathcal{F}_{2}$ is not given by a fibration over $S^{1}$.

We will say that a non-singular curve $\gamma: I \rightarrow M$ describes $\mathcal{F}_{1}$ at $p$ if $p \in \gamma(I)$ and $\gamma$ lies on the leaf of $\mathcal{F}_{1}$ passing through $p$. A point $p \in M$ will be called polynomial (for $\mathcal{F}_{1}$ ) if there exists a curve $\gamma$, describing $\mathcal{F}_{1}$ at $p$, which is polynomial in affine coordinates. The set $P$ of all polynomial points is open and saturated for $\mathcal{F}_{2}$.

THEOREM 1. Let $H$ be a leaf of $\mathcal{F}_{2}$ with non-trivial holonomy. Then the holonomy of $H$ is linear and $H \subset P$.

Corollary 1.1. Assume that $\mathcal{F}_{2}$ has non-trivial holonomy. Then:

(I) $\mathcal{F}_{2}$ has a finite number of minimal sets, all of them with non-trivial holonomy.

(II) $P=M$.

First we deduce Corollary 1.1 from Theorem 1. The number of exceptional minimal sets of $\mathcal{F}_{2}$ is finite and the union of all its compact leaves is a closed set (for $\operatorname{codim} \mathcal{F}_{2}=1$ ). By Theorem 1 the holonomy of each leaf is linear so there are a finite number of compact leaves, all of them with non-trivial holonomy. By Sacksteder's theorem each exceptional minimal set contains a leaf with non-trivial holonomy (see [8]). This proves (I).

On the other hand by Theorem 1 again, $P$ contains every leaf with non-trivial holonomy, so it contains all the minimal sets. Therefore $P=M$; otherwise we could find a minimal set on $\partial P$. This proves (II).

Now for proving Theorem 1 we will study the holonomy of $\mathcal{F}_{2}$ referred to $\mathcal{F}_{1}$.

Consider a point $p \in M$. Let $H_{i}, i=1,2$, be the leaf of $\mathcal{F}_{i}$ passing through $p$ and let $\tau$ be a loop at $p$ on $H_{2}$. In affine coordinates, around $p$, the holonomy map $\varphi_{\tau}$ associated to $\tau$, referred to the transversal $H_{1}$, is given by the restriction of an affine transformation $A$ of $\mathbf{R}^{n}$ respecting the orientation and locally sending $H_{1}$ on $H_{1}$; moreover $A(p)=p$.

Indeed, first consider the case of an arc $\tau^{\prime}$ on $H_{2}$ contained in a convex affine coordinate domain. As $\mathcal{F}_{1}$ and $\mathcal{F}_{2}$ are T.F., its holonomy map $\varphi_{\tau^{\prime}}$, referred to the leaves of $\mathcal{F}_{1}$ passing through the ends of $\tau^{\prime}$, is the restriction of a translation of $\mathbf{R}^{n}$. Now divide $\tau$ into small pieces contained each of them in a convex affine coordinate domain. 
Consider an affine coordinate system with $p$ as origin, i.e. $p \equiv 0$. In this case $A$ is a linear transformation of $\mathbf{R}^{n}$. On the other hand, if $\gamma$ describes $\mathcal{F}_{1}$ at $p$ and $\gamma(0)=p$ then there exist two open intervals $I^{\prime}$ and $I^{\prime \prime}$, containing zero, and a diffeomorphism $\varphi: I^{\prime} \rightarrow I^{\prime \prime}$ such that $\varphi(0)=0$ and $A \circ \gamma=\gamma \circ \varphi$ on $I^{\prime}$.

Deriving with respect to $t$ yields $A\left(\gamma^{\prime}(0)\right)=\varphi^{\prime}(0) \cdot \gamma^{\prime}(0)$, i.e. $\gamma^{\prime}(0)$ is an eigenvector of $A$ with eigenvalue $\varphi^{\prime}(0)>0$ since $\mathcal{F}_{2}$ is transversely orientable.

Proposition 1. If $\varphi_{\tau} \neq$ Id then there exist an eigenspace $W$ of $\mathbf{R}^{n}$, a basis $\left\{e_{1}, \ldots, e_{k}\right\}$ of it, natural numbers $1=r_{1}<r_{2}<\ldots<r_{k}$ and a positive real $\lambda \neq 1$ such that:

(I) $A e_{j}=\lambda^{r_{j}} e_{j}, j=1, \ldots k$.

(II) The curve $\gamma(t)=\sum_{j=1}^{k} t^{r_{j}} e_{j}$ describes $\mathcal{F}_{1}$ at $p$ and $p=\gamma(0)$.

Moreover $\varphi_{\tau}(\gamma(t))=\gamma(\lambda t)$, i.e. the map $\varphi_{\tau}$ is linear.

Rem ark. The isomorphism $A$ regarded as a linear transformation of $T_{p} M$ belongs to the holonomy group, at $p$, of the affine connection.

On the other hand, if $\widetilde{\tau}$ is another loop at $p$ on $H_{2}$ then $\varphi_{\widetilde{\tau}}(\gamma(t))=\gamma(\widetilde{\lambda} t)$ because $\varphi_{\widetilde{\tau}}(\gamma(t))=\widetilde{A}(\gamma(t))$ for some $\widetilde{A} \in G L\left(\mathbf{R}^{n}\right)$. Therefore the holonomy of each leaf of $\mathcal{F}_{2}$ is linear.

It is clear that Theorem 1 follows from Proposition 1. For proving this last result we shall examine all the possible cases.

First case: The real Jordan canonical form of $A$ only has one block. Then there exists a basis $\left\{e_{1}, \ldots, e_{n}\right\}$ of $\mathbf{R}^{n}$ such that $A e_{j}=\lambda e_{j}+e_{j-1}, j=2, \ldots n$, and $A e_{1}=\lambda e_{1}$. Naturally $\lambda=\varphi^{\prime}(0)$. It will be shown that near the origin $\gamma$ lies on the line $\mathbf{R}\left\{e_{1}\right\}$. We will do it for $t \geq 0$; the other side is analogous.

A point $t_{0} \in I^{\prime}$ is called stationary if $\varphi\left(t_{0}\right)=t_{0}$. When $\lambda \neq 1$ the only stationary point close to $0 \in I^{\prime}$ is the zero itself (mean value theorem).

LEMma 1. If $t_{0}>0$ is stationary then $\gamma\left(\left[0, t_{0}\right]\right) \subset \mathbf{R}\left\{e_{1}\right\}$.

Proof. The map $\varphi:\left[0, t_{0}\right] \rightarrow\left[0, t_{0}\right]$ is a diffeomorphism and $A^{k} \circ \gamma=\gamma \circ \varphi^{k}$ where $\varphi^{k}=\varphi \circ \ldots \circ \varphi$. Set $\gamma(t)=\sum_{j=1}^{n} \gamma_{j}(t) e_{j}$. If $\gamma_{n}(t) \neq 0$ for some $t \in\left[0, t_{0}\right]$ then $\lambda=1$ since otherwise $A^{k}(\gamma(t))=\sum_{j=1}^{n-1} f_{j k}(t) e_{j}+\lambda^{k} \gamma_{n}(t) e_{n}$ tends to infinity (if $\lambda<1$ take negative $k)$ and the set $\gamma_{n}\left(\left[0, t_{0}\right]\right)$ is not compact.

But if $\lambda=1$ the $(n-1)$-th coordinate of $A^{k}(\gamma(t))$ equals $\gamma_{n-1}(t)+k \gamma_{n}(t)$ which again tends to infinity unless $\gamma_{n}(t)=0$. In other words $\gamma_{n}=0$. Now the same reasoning shows that $\gamma_{n-1}=\ldots=\gamma_{2}=0$.

By replacing $A$ and $\varphi$ with $A^{-1}$ and $\varphi^{-1}$ respectively if necessary, Lemma 1 allows us to suppose $\varphi(t)<t$ for any $t>0$. Therefore $0<\lambda \leq 1$ and $\lim _{k \rightarrow \infty}\left\{\varphi^{k}(t)\right\}=0$.

1.a) First assume $\lambda=1$. The $(n-1)$-th coordinate of $A^{k}(\gamma(t))$ equals $\gamma_{n-1}(t)+k \gamma_{n}(t)$ which tends to infinity etc... In short $\gamma_{2}=\ldots=\gamma_{n}=0$.

1.b) Now assume $0<\lambda<1$.

Lemma 2. Let $g$ be a function defined around zero such that $g(\varphi(t))=\lambda g(t)$. If $g(0)=g^{\prime}(0)=0$ then $g(t)=0$ for any $t>0$ close to zero. 
Proof. There exist $t_{0}>0$, a constant $B>0$ and a positive integer number $k$ such that $\left|\lambda^{-k}\left(\varphi^{k}\right)^{\prime}(t)\right| \leq B$ for all $t \in\left[0, t_{0}\right]$. Indeed, as $\varphi^{\prime}(0)=\lambda<1$ we can find $0<\lambda^{\prime}<1$ and $t_{0}>0$ such that $\varphi(t) \leq \lambda^{\prime} t$ on $\left[0, t_{0}\right]$. Therefore $\varphi^{k}(t) \leq\left(\lambda^{\prime}\right)^{k} t$.

Set $\mu=\left|\max \left\{\varphi^{\prime \prime}(t) \mid t \in\left[0, t_{0}\right]\right\}\right|$. Then

$$
\left|\varphi^{\prime}\left(\varphi^{k-1}(t)\right)\right| \leq\left|\varphi^{\prime}(0)\right|+\mu \varphi^{k-1}(t) \leq \lambda+\mu \cdot\left(\lambda^{\prime}\right)^{k-1} t
$$

Hence

$$
\begin{aligned}
\left|\lambda^{-k}\left(\varphi^{k}\right)^{\prime}(t)\right| & =\left|\lambda^{-1} \varphi^{\prime}\left(\varphi^{k-1}(t)\right)\right| \cdot\left|\lambda^{1-k}\left(\varphi^{k-1}\right)^{\prime}(t)\right| \\
& \leq\left|1+\mu \lambda^{-1}\left(\lambda^{\prime}\right)^{k-1} t\right| \cdot\left|\lambda^{1-k}\left(\varphi^{k-1}\right)^{\prime}(t)\right| \leq \ldots \\
& \leq \prod_{j=1}^{k-1}\left(1+\mu \lambda^{-1}\left(\lambda^{\prime}\right)^{j} t\right)\left|\lambda^{-1} \varphi^{\prime}(t)\right| \leq \exp \left(\mu \lambda^{-1} \lambda^{\prime}\left(1-\lambda^{\prime}\right)^{-1} t\right)\left|\lambda^{-1} \varphi^{\prime}(t)\right| \leq B .
\end{aligned}
$$

On the other hand $g(t)=\lambda^{-k} g\left(\varphi^{k}(t)\right)$ when $t \in\left[0, t_{0}\right]$; so

$$
\left|g^{\prime}(t)\right| \leq\left|\lambda^{-k}\left(\varphi^{k}\right)^{\prime}(t)\right| \cdot\left|g^{\prime}\left(\varphi^{k}(t)\right)\right| \leq B\left|g^{\prime}\left(\varphi^{k}(t)\right)\right| \rightarrow 0,
$$

because $\left\{\varphi^{k}(t)\right\} \rightarrow 0$ and $g^{\prime}(0)=0$. Therefore $g=0$ on $\left[0, t_{0}\right]$, since $g(0)=0$.

Consider the curve $\gamma$ again. If $n \geq 2$ then $\gamma_{2}^{\prime}(0)=\ldots=\gamma_{n}^{\prime}(0)=0$ as $\gamma^{\prime}(0)$ is an eigenvector. Moreover $\gamma_{n}(\varphi(t))=\lambda \gamma_{n}(t)$ for $A(\gamma(t))=\gamma(\varphi(t))$, whence $\gamma_{n}=0$. But then $\gamma_{n-1}(\varphi(t))=\lambda \gamma_{n-1}(t)$ etc. . To sum up $\gamma_{2}=\ldots=\gamma_{n}=0$.

Finally by changing the parametrization of the curve if necessary, we may suppose $\gamma(t)=t e_{1}$.

Second case: The real Jordan canonical form of $A$ has two or more blocks. Consider a decomposition $V=\bigoplus_{\ell=1}^{m} V^{\ell}$ where each $V^{\ell}$ is an eigenspace and each linear map $A_{\mid V^{\ell}}$ has one block only.

Set $\gamma=\left(\gamma^{1}, \ldots, \gamma^{m}\right)$. Then every component $\left(\gamma^{\ell}\right)^{\prime}(0)$ is an eigenvector of $A_{\mid V^{\ell}}$ and at least one of them does not vanish, for example $\left(\gamma^{1}\right)^{\prime}(0)$. The first case applied to $\gamma^{1}$ and $A_{\mid V^{1}}$, allows us to find a basis $\left\{e_{1}^{1}, \ldots, e_{n_{1}}^{1}\right\}$ of $V^{1}$ and a parametrization of $\gamma$ such that $A e_{j}^{1}=\lambda_{1} e_{j}^{1}+e_{j-1}^{1}, j=2, \ldots, n_{1}, A e_{1}^{1}=\lambda_{1} e_{1}^{1}, \gamma^{1}(t)=t e_{1}^{1}$ and $\varphi(t)=\lambda_{1} t$.

If $\lambda_{1}=1$ then $\varphi_{\tau}=$ Id. Therefore assume $0<\lambda_{1}<1$ (if $\lambda_{1}>1$ take $A^{-1}$ and $\varphi^{-1}$ instead of $A$ and $\varphi$ ).

First consider the subspaces $V^{j}$ such that $A_{\mid V^{j}}$ has a real eigenvalue. For the sake of simplicity suppose that it is the case of $V^{2}$. Choose a basis $\left\{e_{1}^{2}, \ldots, e_{n_{2}}^{2}\right\}$ such that $A e_{j}^{2}=\lambda_{2} e_{j}^{2}+e_{j-1}^{2}, j=2, \ldots n_{2}$, and $A e_{1}^{2}=\lambda_{2} e_{1}^{2}$. Set $\gamma^{2}=\sum_{j=1}^{n_{2}} h_{n_{2}-j} e_{j}^{2}$. Then $\lambda_{2} h_{0}(t)=$ $h_{0}\left(\lambda_{1} t\right)$ as $A \circ \gamma=\gamma \circ \varphi$. So $h_{0}(t)=\lambda_{2}^{-r} h_{0}\left(\lambda_{1}^{r} t\right)$ and $h_{0}^{(k)}(t)=\lambda_{1}^{k r} \lambda_{2}^{-r} h_{0}^{(k)}\left(\lambda_{1}^{r} t\right)$.

From some positive integer number on $\left|\lambda_{1}^{k} \lambda_{2}^{-1}\right|<1$, and $h_{0}^{(k)}(t)=0$ because $\left\{\lambda_{1}^{r} t\right\} \rightarrow$ 0 . In other words, $h_{0}$ is a polynomial.

On the other hand $h_{0}^{(s)}(0)=\lambda_{1}^{s} \lambda_{2}^{-1} h_{0}^{(s)}(0)$. If $\lambda_{2}$ is not a positive power of $\lambda_{1}$ then $h_{0}=0$. Doing the same with $h_{1}$, then with $h_{2}$ and so on, yields $h_{0}=h_{1}=\ldots=h_{n_{2}-1}=$ 0; i.e. $\gamma^{2}=0$.

If $\lambda_{2}=\lambda_{1}^{k}$, where $k \in \mathbf{N}-\{0\}$, then $h_{0}(t)=a t^{k}$. Moreover $h_{1}\left(\lambda_{1} t\right)=\lambda_{2} h_{1}(t)+$ $h_{0}(t)$ since $A \circ \gamma=\gamma \circ \varphi$. Hence $h_{1}^{(k+1)}(t)=\lambda_{1}^{k+1} \lambda_{2}^{-1} h_{1}^{(k+1)}\left(\lambda_{1} t\right)=\lambda_{1} h_{1}^{(k+1)}\left(\lambda_{1} t\right)$ and $h_{1}^{(k+1)}(t)=\lambda_{1}^{r} h_{1}^{(k+1)}\left(\lambda_{1}^{r} t\right)$. Therefore $h_{1}^{(k+1)}=0$ because $\left\{\lambda_{1}^{r}\right\} \rightarrow 0$. 
In a word $h_{1}(t)=\sum_{j=0}^{k} b_{j} t^{j}$. Now the relation $h_{1}\left(\lambda_{1} t\right)=\lambda_{2} h_{1}(t)+h_{0}(t)$ implies that $h_{0}=0$ and $h_{1}(t)=b_{k} t^{k}$.

By a similar argument $h_{1}=\ldots=h_{n_{2}-2}=0$ and $\gamma^{2}(t)=c t^{k} e_{1}^{2}$.

For the other blocks with real eigenvalues we do the same. If $A_{\mid V^{\ell}}$ has no real eigenvalue by complexifying it we obtain two blocks with non-real eigenvalues $\lambda^{\prime}$ and $\bar{\lambda}^{\prime}$ respectively. Obviously $\lambda^{\prime}$ and $\bar{\lambda}^{\prime}$ are not powers of $\lambda_{1}$ therefore $\gamma^{\ell}=0$.

By rearranging according to powers of $t$ we obtain a family $\left\{e_{1}, \ldots, e_{k}\right\}$ of eigenvectors, with eigenvalues $\lambda^{r_{j}}, j=1, \ldots k$, where $\lambda=\lambda_{1}$ and $1=r_{1}<r_{2}<\ldots<r_{k}$, such that $\gamma(t)=\sum_{j=1}^{k} t^{r_{j}} e_{j}$. That completes the proof of Proposition 1 .

3. The degree of flatness of $\mathcal{F}_{1}$. Given a curve $\gamma$ on $M$, by definition $\gamma^{(1)}$ is its velocity, $\gamma^{(2)}$ its acceleration, i.e. the covariant derivative of $\gamma^{(1)}$ along $\gamma, \gamma^{(3)}$ the covariant derivative of $\gamma^{(2)}$ along $\gamma$ etc... In affine coordinates $\gamma^{(k)}$ is just the $k$-th derivative of $\gamma$ with respect to the parameter. The maximum number of linearly independent successive derivatives $\gamma^{(1)}\left(t_{0}\right), \gamma^{(2)}\left(t_{0}\right), \ldots, \gamma^{(k)}\left(t_{0}\right)$ at a point $p=\gamma\left(t_{0}\right)$ does not depend on the parametrization. When $\gamma$ describes $\mathcal{F}_{1}$ at $p$ we denote this number by $s(p)$. That defines a locally increasing function $s: M \rightarrow \mathbf{N}$ which is constant along the leaves of $\mathcal{F}_{2}$ since $\mathcal{F}_{1}$ and $\mathcal{F}_{2}$ are T.F.

Lemma 3. Suppose that $s: M \rightarrow \mathbf{N}$ is constant and set $k=s(M)$. Then a curve $\gamma$ describing $\mathcal{F}_{1}$, at a point, locally lies on a well defined affine $k$-plane; i.e. if we identify to each other an open set of $M$ and one of $\mathbf{R}^{n}$ by means of an affine system of coordinates, then $\gamma$ is locally contained just in an affine $k$-plane of $\mathbf{R}^{n}$.

Continue to suppose $k=s(M)$. Let $\mathcal{G}$ be the vector subbundle of $T M$ whose fibre at $p$ is the subspace spanned by $\gamma^{(1)}\left(t_{0}\right), \gamma^{(2)}\left(t_{0}\right), \ldots, \gamma^{(k)}\left(t_{0}\right)$ when $\gamma$ describes $\mathcal{F}_{1}$ at $p=\gamma\left(t_{0}\right)$. By Lemma 3, as $\mathcal{F}_{1}$ and $\mathcal{F}_{2}$ are T.F., around each point there exist affine coordinates $\left(x_{1}, \ldots, x_{n}\right)$ on which $\mathcal{G}$ is defined by $d x_{k+1}=\ldots=d x_{n}=0$. Therefore $\mathcal{G}$ is parallel and the foliation associated to it contains $\mathcal{F}_{1}$. Besides $d x_{1} \wedge \ldots \wedge d x_{k}$ locally defines a volume form $\omega$, on $\mathcal{G}$, parallel as well. This form $\omega$ does not depend on the choice of the affine coordinate system up to a constant factor.

Set $f(t)=\omega\left(\gamma^{(1)}(t), \ldots, \gamma^{(k)}(t)\right)$ and $\tau(u)=\gamma(\lambda(u))$. Then $\omega\left(\tau^{(1)}(u), \ldots, \tau^{(k)}(u)\right)=$ $\left(\lambda^{\prime}(u)\right)^{\ell} f(\lambda(u))$ where $\ell=k(k+1) / 2$. Therefore for each $p \in M$ there exists a curve that we call $\gamma$ again, describing $\mathcal{F}_{1}$ at this point, such that $\omega\left(\gamma^{(1)}(t), \ldots, \gamma^{(k)}(t)\right)$ is constant. Obviously this property does not depend on the choice of $\omega$. Moreover if $\omega\left(\tau^{(1)}(u), \ldots, \tau^{(k)}(u)\right)$ is constant as well, where $\tau(u)=\gamma(\lambda(u))$, then $\left(\lambda^{\prime}(u)\right)^{\ell}$ is constant and $\lambda$ has to be an affine function of $u$. In other words the relation between two of these parametrizations is given by an affine transformation of $\mathbf{R}$. Therefore $\mathcal{F}_{1}$ is equipped with an affine structure.

By construction this affine structure is preserved by translation along the leaves of $\mathcal{F}_{2}$ because $\omega$ is parallel. Consequently, as $\mathcal{F}_{1}$ and $\mathcal{F}_{2}$ are T.F., we have:

THEOREM 2. If $s: M \rightarrow \mathbf{N}$ is constant then $\mathcal{F}_{2}$ has a transverse affine structure.

Now suppose that each leaf of $\mathcal{F}_{2}$ is dense and has trivial holonomy. Then $s(M)=k$ for some $k \in \mathbf{N}-\{0\}$ and $\mathcal{F}_{2}$ has a transverse affine structure. By Seke's result (see 
Theorem 8 of [9]), $\mathcal{F}_{2}$ is defined by a non-singular closed form $\alpha$. Let $X$ be the vector field tangent to $\mathcal{F}_{1}$ such that $\alpha(X)=1$. Obviously $X$ is $\mathcal{F}_{2}$-foliate.

Lemma 4. Let $X$ be an $\mathcal{F}_{2}$-foliate vector field tangent to $\mathcal{F}_{1}$; then $X$ is parallel along $\mathcal{F}_{2}$. Moreover, if $Y$ is parallel along $\mathcal{F}_{2}$ so is $\nabla_{X} Y$.

By Lemma 4 each vector field $X_{j}=\nabla_{X}^{j} X$ is $\mathcal{F}_{2}$-parallel; so $\nabla_{X}^{k} X=\sum_{j=0}^{k-1} a_{j} X_{j}$, where $a_{j} \in \mathbf{R}, j=0, \ldots, k-1$, since $s(M)=k$.

Set $T M=T \mathcal{F}_{1} \oplus T \mathcal{F}_{2}$ and let $\varphi$ be the projection onto $T \mathcal{F}_{2}$. Given vector fields $Z_{1}$ and $Z_{2}$ tangent to $\mathcal{F}_{2}$ set, by definition, $\nabla_{Z_{1}}^{\prime} Z_{2}=\varphi\left(\nabla_{Z_{1}} Z_{2}\right)$. As $\mathcal{F}_{1}$ and $\mathcal{F}_{2}$ are T.F. it is easily seen that $\nabla^{\prime}$ is a connection on the leaves of $\mathcal{F}_{2}$ whose torsion and curvature vanish.

On the other hand, as each $X_{j}$ is $\mathcal{F}_{2}$-parallel and $\mathcal{F}_{1}$ and $\mathcal{F}_{2}$ are T.F., it yields $\nabla^{\prime} \varphi\left(X_{j}\right)=0$; so $\left[\varphi\left(X_{j}\right), \varphi\left(X_{\ell}\right)\right]=0$. But $\varphi\left(X_{1}\right), \ldots, \varphi\left(X_{k-1}\right)$ are linearly independent because $s(M)=k$. Therefore $k \leq \operatorname{rank} M+1$ (we recall that the rank of a compact manifold is the maximum number of commuting vector fields linearly independent everywhere). For example if $\mathcal{F}_{1}$ is as much twisted as possible, i.e. $s(M)=n$, then $\operatorname{rank} M \geq n-1$ and $M$ is a bundle over $S^{1}$ with fibre $\mathbf{T}^{n-1}$ (see [2]).

In short:

TheOREm 3. If every leaf of $\mathcal{F}_{2}$ is dense and has trivial holonomy then there exist $k \in \mathbf{N}-\{0\}, a_{0}, \ldots, a_{k-1} \in \mathbf{R}$ and a non-singular vector field $X$ such that:

(a) $s(M)=k \leq \operatorname{rank} M+1$.

(b) $X$ is tangent to $\mathcal{F}_{1}, \mathcal{F}_{2}$-parallel and $\mathcal{F}_{2}$-foliate; therefore $\mathcal{F}_{2}$ is given by a nonsingular closed 1-form.

(c) $\nabla_{X}^{k} X=\sum_{j=0}^{k-1} a_{j} \nabla_{X}^{j} X$.

Moreover $X$ is unique up to a constant factor.

Let us remark that in affine coordinates $\mathcal{F}_{1}$ is described by a solution of the equation

$$
\gamma^{(k+1)}=\sum_{j=1}^{k} a_{j-1} \gamma^{(j)}
$$

therefore its shape is completely known.

ExAmple. Consider the torus $\mathbf{T}^{n}$ equipped with the canonical affine structure. Then $\mathcal{F}_{2}$ has trivial holonomy (see the remark following Proposition 1).

As the slope of $\mathcal{F}_{1}$ along each leaf of $\mathcal{F}_{2}$ is constant, if all the leaves of this last foliation are dense then the vector field given by Theorem 3 is geodesic, i.e. $X=\sum_{j=1}^{n} b_{j} \frac{\partial}{\partial \theta_{j}}$ where $b_{1}, \ldots, b_{n} \in \mathbf{R}$. On the other hand $\mathcal{F}_{2}$ is defined by a closed 1 -form $\alpha=\sum_{j=1}^{n} b_{j}^{\prime} d \theta_{j}+\alpha^{\prime}$, where $b_{1}^{\prime}, \ldots, b_{n}^{\prime} \in \mathbf{R}$ with $\sum_{j=1}^{n} b_{j} b_{j}^{\prime}=1$ and $\alpha^{\prime}$ is the pull-back of a closed 1 -form defined on the quotient of $\mathbf{T}^{n}$ by the closures of the orbits of $X$.

If all the leaves of $\mathcal{F}_{2}$ are compact, consider an embedded curve $\tau: S^{1} \rightarrow M$ transverse to $\mathcal{F}_{2}$ and cutting each of its leaves once. Then $\mathcal{F}_{1}$ is describes by its value on $\tau$. Indeed given a vector field $X^{\prime}$ along $\tau$, transverse to $\mathcal{F}_{2}$, the parallel translation along the leaves of this foliation gives rise to a vector field $X$ on $\mathbf{T}^{n}$, which defines a foliation $\mathcal{F}_{1}$. Suppose that $X$ is never tangent to $\mathcal{F}_{2}$; then $\mathcal{F}_{1}$ and $\mathcal{F}_{2}$ are T.F. if and only if $X$ is $\mathcal{F}_{2}$-foliate. 
4. The non-trivial holonomy case. In this section we assume that $\mathcal{F}_{2}$ has at least a leaf with non-trivial holonomy. Given natural numbers $j_{1}=1<j_{2}<\ldots<j_{\ell}$ let $M_{1 j_{2} \ldots j_{\ell}}$ be the set of all the points $p \in M$ for which the following property holds: there exist $t_{0} \in \mathbf{R}$ and an affine coordinate system, defined around $p$, such that the curve $\gamma(t)=\left(t, t^{j_{2}}, \ldots, t^{j_{\ell}}, 0, \ldots, 0\right)$ describes $\mathcal{F}_{1}$ at $\gamma\left(t_{0}\right)=p$. By construction $M_{1 j_{2} \ldots j_{\ell}}$ is an $\mathcal{F}_{2}$-saturated open set. Besides $\partial M_{1 j_{2} \ldots j_{\ell}}=\emptyset$, i.e. either $M_{1 j_{2} \ldots j_{\ell}}=\emptyset$ or $M_{1 j_{2} \ldots j_{\ell}}=M$.

Indeed, if $\partial M_{1 j_{2} \ldots j_{\ell}} \neq \emptyset$ it contains a minimal set. Therefore, by reasoning as before, the boundary of $M_{1 j_{2} \ldots j_{\ell}}$ contains a leaf $H$ of $\mathcal{F}_{2}$ with non-trivial holonomy. By Proposition 1 there exist natural numbers $r_{1}=1<r_{2}<\ldots<r_{k}$ such that $H \subset M_{1 r_{2} \ldots r_{k}}$.

Lemma 5. Consider, on $\mathbf{R}^{n}$, the curves $\gamma(t)=\left(t, t^{j_{2}}, \ldots, t^{j_{\ell}}, 0, \ldots, 0\right)$ and $\lambda(t)=\left(t, t^{r_{2}}, \ldots, t^{r_{k}}, 0, \ldots, 0\right)$ where $1<j_{2}<\ldots<j_{\ell}$ and $1<r_{2}<\ldots<r_{k}$. Suppose that there exists an affine transformation $A: \mathbf{R}^{n} \rightarrow \mathbf{R}^{n}$ and a non-empty open interval $I$ such that $A(\gamma(I)) \subset \lambda(\mathbf{R})$. Then $\ell=k$ and $j_{i}=k_{i}, i=2, \ldots, \ell$.

Now Lemma 5 says us that $H \subset M_{1 j_{2} \ldots j_{\ell}}$ so $H \cap \partial M_{1 j_{2} \ldots j_{\ell}}=\emptyset$, contradiction.

We have assumed that $\mathcal{F}_{2}$ has non-trivial holonomy; therefore by Proposition 1 there exist natural numbers $r_{1}=1<r_{2}<\ldots<r_{k}$, a point $p_{0} \in M_{1 r_{2} \ldots r_{k}}$ and an affine coordinate system, defined around this one, such that the curve $\gamma(t)=\left(t, t^{r_{2}}, \ldots, t^{r_{k}}, 0, \ldots, 0\right)$ describes $\mathcal{F}_{1}$ at $p_{0}=\gamma(0)$. Moreover $M=M_{1 r_{2} \ldots r_{k}}$.

First case: Function $s: M \rightarrow \mathbf{N}$ is constant. Then $s(M)=k$ and $r_{i}=i, i=2, \ldots, k$ since $p_{0}=\gamma(0)$ for some $p_{0}$. It is easily seen that given $a \in \mathbf{R}^{+}$and $b \in \mathbf{R}$ there exists an affine transformation $A$ of $\mathbf{R}^{n}$, preserving the orientation such that $A(\gamma(t))=$ $\gamma(a t+b)$, i.e. all the points of $\gamma$ are affinely equivalent on $\mathbf{R}^{n}$. Therefore for each $p \in M$ we can find affine coordinates, defined around this point, on which the curve $\gamma(t)=$ $\left(t, t^{2}, \ldots, t^{k}, 0, \ldots, 0\right)$ describes $\mathcal{F}_{1}$ at $\gamma(0)=p$.

In Example (d) and the second part of Example (f) (set $r_{j}=j$ ), $\mathcal{F}_{2}$ has one minimal set if $n \geq 3$ and two minimal sets if $n=2$; they are compact leaves. In Example (g) the only minimal set of $\mathcal{F}_{2}$ is $M$ itself.

On the other hand, as $\mathcal{F}_{2}$ has a transverse affine structure (Theorem 2), if the fundamental group of $M$ is Abelian then the minimal sets of $\mathcal{F}_{2}$ are just the compact leaves (see [4] and [9]).

Second case: Function $s: M \rightarrow \mathbf{N}$ is not constant. Consider the curve $\gamma(t)=\left(t, t^{r_{2}}, \ldots, t^{r_{k}}, 0, \ldots, 0\right)$ and for each $a \in \mathbf{R}-\{0\}$ the affine transformation of $\mathbf{R}^{n}$ : $A_{a}(x)=\left(a x_{1}, a^{r_{2}} x_{2}, \ldots, a^{r_{k}} x_{k}, x_{k+1}, \ldots, x_{n}\right)$. Then $A_{a}(\gamma(t))=\gamma(a t)$; so all the points of $\gamma$, unless the origin, are affinely equivalent and they have the same number of linearly independent successive derivatives, which equals $k$. Set $M^{\prime}=s^{-1}(k)$. Then:

1) $k<r_{k}$, and $s(p)<k$ if $p=\gamma(0)$. Moreover $s\left(M-M^{\prime}\right)$ is the first natural number $i$ such that $i+1<r_{i+1}$.

2) $M-M^{\prime}$ is transversely finite; therefore it is the union of a finite number of compact leaves of $\mathcal{F}_{2}$, each of them with non-trivial holonomy (Corollary 1.1).

3) The leaves of $\mathcal{F}_{2}$ contained in $M^{\prime}$ have trivial holonomy (because $s(p)<k$ if $p=\gamma(0))$ and $M^{\prime}$ does not contain any minimal set. 
Besides around every point $p \in M$ we can find affine coordinates on which the curve $\gamma(t)=\left(t, t^{r_{2}}, \ldots, t^{r_{k}}, 0, \ldots, 0\right)$ describes $\mathcal{F}_{1}$ at $p$, where $p=\gamma(1)$ if $p \in M^{\prime}$ and $p=\gamma(0)$ if $p \notin M^{\prime}$.

Finally remark that the affine transformations of $\mathbf{R}^{n}$ sending a non-empty open interval of the curve $\gamma(t)=\left(t, t^{r_{2}}, \ldots, t^{r_{k}}, 0, \ldots, 0\right)$ on a subset of $\gamma(\mathbf{R})$ are the transformations $A_{a}$ defined before. As $A_{a}(\gamma(t))=\gamma(a t)$ the parameter $t$ gives rise to an affine structure on each leaf of $\mathcal{F}_{1}$, and a transverse affine structure of $\mathcal{F}_{2}$ because $\mathcal{F}_{1}$ and $\mathcal{F}_{2}$ are T.F. Moreover the holonomy of this affine transverse structure is a group of homotheties with the same center. This implies that on each connected component of $M^{\prime}$ either all the leaves of $\mathcal{F}_{2}$ are locally dense or all of them are proper. Even more this proves, in another way, that $\mathcal{F}_{2}$ has almost no holonomy, i.e. only the compact leaves have non-trivial holonomy (Theorem 7 of [9], see [4] as well).

In short:

THEOREM 4. If $\mathcal{F}_{2}$ has non-trivial holonomy and function $s: M \rightarrow \mathbf{N}$ is not constant then:

(I) $s(M)=\left\{\ell_{1}, \ell_{2}\right\}$ where $\ell_{1}<\ell_{2}$.

(II) $\mathcal{F}_{2}$ has a transverse affine structure whose holonomy is group of homotheties of $\mathbf{R}$ with a common center.

(III) $\mathcal{F}_{2}$ has almost no holonomy; moreover $s^{-1}\left(\ell_{1}\right)$ is the union of all compact leaves of this foliation (a finite number).

Although there exist codimension one foliations with a transverse affine structure and exceptional minimal sets (see [6]), I do not know any example of translation foliations where $\mathcal{F}_{2}$ has an exceptional minimal set. Obviously in such a case $s: M \rightarrow \mathbf{N}$ has to be constant.

ExAmple. Suppose that $M=S^{1} \times S^{m}, m \geq 2$. Then $\mathcal{F}_{2}$ has non-trivial holonomy and its minimal sets are compact leaves.

Indeed, by Theorem 3 if $\mathcal{F}_{2}$ has trivial holonomy, as $H^{1}\left(S^{1} \times S^{m}, \mathbf{R}\right)=\mathbf{R}$, then $\mathcal{F}_{2}$ is defined by a fibration $\pi: S^{1} \times S^{m} \rightarrow S^{1}$. Therefore each leaf of $\mathcal{F}_{2}$ is simply connected because the homotopy sequence. On the other hand (see Section 3 ) every leaf has an affine structure and, by parallel displacement, we may construct a parallel non-singular 1-form $\alpha$ on it. Obviously $d \alpha=0$. So $[\alpha] \neq 0$ and the leaf is not simply connected, contradiction.

A transverse affine structure $\mathcal{S}$ of $\mathcal{F}_{2}$ gives rise, through the local $\mathcal{F}_{2}$-foliate vector fields which are tangent to $\mathcal{F}_{1}$, to an affine structure on each leaf of $\mathcal{F}_{1}$. When all these structures are complete we will say that $\mathcal{S}$ is complete (with respect to $\mathcal{F}_{1}$ ).

Let us call $\nabla(\mathcal{S})$ the connection on $\mathcal{F}_{1}$ associated to $\mathcal{S}$.

For example in the case of Theorem 3 the transverse affine structure $\mathcal{S}_{1}$ associated to $X$ is complete because $\nabla\left(\mathcal{S}_{1}\right)_{X} X=0$. On the other hand the transverse affine structure $\mathcal{S}_{2}$ built up from the property that $s(M)=k$ is complete iff $a_{k-1}=0$, i.e. if and only if $\mathcal{S}_{1}=\mathcal{S}_{2}$. 
THEOREM 5. Assume that $\mathcal{F}_{2}$ has a complete transverse affine structure $\mathcal{S}$. If the holonomy of $\mathcal{F}_{2}$ is not trivial then all its leaves are dense (therefore the function $s: M \rightarrow$ $\mathbf{N}$ is constant).

Proof. Let $\widetilde{M}$ be the universal covering of $M$. Then the structure $\left(M, \mathcal{F}_{1}, \mathcal{F}_{2}\right)$ can be seen as the quotient of a structure $\left(\widetilde{M}, \widetilde{\mathcal{F}}_{1}, \widetilde{\mathcal{F}}_{2}\right)$ of the same kind (i.e. $\widetilde{M}$ is an affine manifold and $\widetilde{\mathcal{F}}_{1}$ and $\widetilde{\mathcal{F}}_{2}$ are T.F.) by the action of a group $G$, isomorphic to the fundamental group of $M$, which operates properly discontinuously. Denote by $\pi: \widetilde{M} \rightarrow M$ the canonical projection. Since $\mathcal{F}_{2}$ is transversely orientable and has a transverse affine structure, there exist an $\widetilde{\mathcal{F}}_{2}$-basic submersion $\varphi: \widetilde{M} \rightarrow \mathbf{R}$ and a morphism $\rho: G \rightarrow \mathrm{Aff}^{+}(\mathbf{R})$ such that $\varphi(g \cdot p)=\rho(g) \cdot \varphi(p)$ for any $g \in G$ and $p \in \widetilde{M}$ (see [4]).

Let $X$ be the vector field on $\widetilde{M}$, tangent to $\mathcal{F}_{1}$, such that $\varphi_{*}(X)=\partial / \partial t$. If $\gamma(t)$ is an integral curve of $X$ then $\pi(\gamma(t))$ is a geodesic of $\nabla(\mathcal{S})$. Therefore $X$ is complete; consequently the fibration $\varphi: \widetilde{M} \rightarrow \mathbf{R}$ is a product and each $\varphi^{-1}(t)$ is a leaf of $\widetilde{\mathcal{F}}_{2}$.

We can suppose, without loss of generality, that the leaf $\pi\left(\varphi^{-1}(0)\right)$ has non-trivial holonomy: so there exists $0<a<1$ such that the map $t \rightarrow a t$ belongs to $\rho(G)$.

First assume that $\rho(G)$ contains some translation. Then for each $t_{0} \in \mathbf{R}$ the set $\rho(G)\left(t_{0}\right)$ is dense. As $\varphi: \widetilde{M} \rightarrow \mathbf{R}$ is a product, $\pi\left(\varphi^{-1}\left(\rho(G)\left(t_{0}\right)\right)\right)$ is a dense leaf of $\mathcal{F}_{2}$. But all the leaves of this foliation can be written in this way, so they are dense.

If $\rho(G)$ does not contain any translation then it is a group of homotheties with center $0 \in \mathbf{R}$. Moreover $\mathcal{F}_{2}$ has only a minimal set: the compact leaf $\pi\left(\varphi^{-1}(0)\right)$, and every leaf of $\mathcal{F}_{1}$ intersects $\pi\left(\varphi^{-1}(0)\right)$ just once. Let us choose the orientation of $\mathcal{F}_{1}$ whose pull-back by $\pi$ equals that given by $X$ on $\widetilde{\mathcal{F}}_{1}$. If $L$ is a leaf of $\mathcal{F}_{1}$ then its $\alpha$-limit is contained in the closed set $\pi\left(\varphi^{-1}([0,+\infty))\right)$. Therefore no leaf of this $\alpha$-limit cuts $\pi\left(\varphi^{-1}(0)\right)$, contradiction.

R e m a rk. We recall that if the holonomy of $\mathcal{F}_{2}$ is not trivial then this foliation at most has one transverse affine structure (see [4] and [9]).

Corollary 5.1. Suppose that the affine manifold $(M, \nabla)$ is complete. If $\mathcal{F}_{2}$ has nontrivial holonomy then each of its leaves is dense (therefore $s: M \rightarrow \mathbf{N}$ is constant).

Pr o of. Now the affine manifold $\widetilde{M}$ can be regarded as $\mathbf{R}^{n}$ endowed with the canonical affine structure. Then every leaf $\widetilde{L}$ of $\widetilde{\mathcal{F}}_{1}$ may be written, in suitable affine coordinates, in the form $\left\{\left(t, t^{r_{2}}, \ldots, t^{r_{k}}, 0, \ldots, 0\right) \mid t \in \mathbf{R}\right\}$. Indeed, $\widetilde{L}$ locally is a pseudo-parabola and it has no ends on this curve because is a leaf.

Let $\mathcal{S}$ be the transverse affine structure of $\mathcal{F}_{2}$ constructed before (see Theorems 2 and 4). Set $\gamma(t)=\left(t, t^{r_{2}}, \ldots, t^{r_{k}}, 0, \ldots, 0\right), t \in \mathbf{R}$. If $\left\{\left(t, t^{r_{2}}, \ldots, t^{r_{k}}, 0, \ldots, 0\right) \mid t \in \mathbf{R}\right\}$ is a leaf of $\widetilde{\mathcal{F}}_{1}$ then it is easily seen that $\pi(\gamma(t))$ is a geodesic of $\nabla(\mathcal{S})$. Therefore $\mathcal{S}$ is complete.

\section{References}

[1] R. Brouzet, P. Molino and F. J. Turiel, Géométrie des systèmes bihamiltoniens, Indag. Math. (N.S.) 4(3) (1993), 269-296.

[2] G. Châtelet and H. Rosenberg, Manifolds which admit $\mathbf{R}^{n}$ actions, Inst. Hautes Études Sci. Publ. Math. 43 (1974), 245-260. 
[3] G. Darboux, Leçons sur la Théorie générale de Surfaces, Gauthier-Villars, Paris.

[4] C. Godbillon, Feuilletages: études géométriques, Progr. Math. 98, Birkhäuser, 1991.

[5] I. M. Gelfand and I. Zakharevich, Webs, Veronese curves, and Bihamiltonian systems, J. Funct. Anal. 99 (1991), 150-178.

[6] G. Hector, Quelques exemples de feuilletages-Espèces rares, Ann. Inst. Fourier (Grenoble) 26(1) (1976), 239-264.

[7] G. Hector and U. Hirsch, Introduction to the Geometry of Foliations. Part B, Aspects Math. E3, Friedr. Vieweg \& Sohn, Braunschweig/Wiesbaden, 1987.

[8] R. Sacksteder, Foliations and pseudo-groups, Amer. J. Math. 87 (1965), 79-102.

[9] B. Seke, Sur les structures transversalement affines des feuilletages de codimension un, Ann. Inst. Fourier (Grenoble) 30(1) (1980), 1-29. 